\title{
Desenvolvimento de competências de gêmeos autistas na pré-escola: relato de caso
}

\author{
Development of competencies de autistic twins in the pré- \\ school: case report
}

\author{
Desarrollo de competencias de gemelos autista en \\ preescolar: reporte de caso
}

\section{Leiliane Tavares de Albuquerque ${ }^{1}$, Daniella kelly Dos Santos Silva ${ }^{2}$, Fausto Muñoz-Lara ${ }^{3}$, Thayane de Deus Branco Nobre ${ }^{4}$, Magnúcia de Lima Leite ${ }^{5}$, Rafael Vinicius de Almeida Tabosa6, Valtuir Barbosa Félix ${ }^{7}$, Sura Amélia Barbosa Félix Leão ${ }^{8}$, Euclides Maurício Trindade-Filho ${ }^{9}$, José Claudio da Silva ${ }^{10}$}

\begin{abstract}
1.Psicóloga no Centro da Primeira Infância Maria Bergmann. Maceió-AL, Brasil. Orcid: https://orcid.org/0000-0001-8111-1391

2.Psicologia, Centro Universitário UNIT. Maceió-AL, Brasil. Orcid: https://orcid.org/0000-0003-00887097

3.Médico. Facultad de Ciencias Medicas, Universidad Nacional Autónoma de Honduras (UNAH), Tegucigalpa, Honduras.

4.Acadêmica de Medicina pelo Centro Universitário CESMAC. Maceió-AL, Brasil. Orcid: https://orcid.org/0000-0002-0107-8649

5.Doutoranda em Saúde Pública na Universidade de São Paulo - USP. Docente na Universidade Estadual de Ciências da Saúde de Alagoas (UNCISAL). Maceió-AL, Brasil. Orcid: https://orcid.org/0000-00025465-9287

6.Acadêmico de Fisioterapia pelo Centro Universitário CESMAC. Maceió-AL, Brasil. Orcid: https://orcid.org/0000-0002-6680-2677

7.Cirurgião Bucomaxilofacial do HUPAA/UFAL/EBSERH. Professor de Cirurgia da FOUFAL/UFAL; Chefe da Divisão de Apoio e Diagnóstico Terapêutico do HUPAA/ UFAL. Maceió-AL, Brasil. Orcid: https://orcid.org/0000-0002-2961-2487

8. Médica especialista na Santa Casa de Misericórdia de Maceió. Docente na Universidade Federal de Alagoas (UFAL), Campus Arapiraca-AL, Brasil. Orcid: https://orcid.org/0000-0003-0944-2246

9.Médico, Doutor. Docente no Centro Universitário CESMAC. Laboratório de Neurociência, Núcleo de Ciências Biológicas (NUCIB), Universidade Estadual de Ciências da Saúde de Alagoas (UNCISAL). Maceió-AL, Brasil. Orcid: https://orcid.org/0000-0001-6819-1673

10.Fisioterapeuta, Doutor. Docente no Centro Universitário CESMAC e no Laboratório de Neurociência, Núcleo de Ciências Biológicas (NUCIB), Universidade Estadual de Ciências da Saúde de Alagoas (UNCISAL). Maceió-AL, Brasil. Orcid: https://orcid.org/0000-0003-3749-2822
\end{abstract}

\section{Resumo}

Introdução. O autismo infantil faz parte de um grupo de transtornos do Neurodesenvolvimento descritos pelo Manual Diagnostico e Estatístico de Transtornos Mentais 5 (DSM-5). As crianças com transtorno autista apresentam comprometimentos funcionais fundamentais. Objetivo. Descrever aspectos do desenvolvimento comportamental em gêmeos (G\#1 e G\#2) univitelinos com diagnóstico médico concordante para autismo, em fase pré-escolar. Método. Estudo de corte transversal, prospectivo-descrito. A coleta de dados foi realizada através dos questionários: CARS - Childhood Autism Rating Scale; Escala de Perfil Psicoeducacional Revisto; Escala de Comportamento Adaptativo Vineland. Resultados. Na CARS, G\#1 foi classificado como não autista e G\#2 um autista moderado. $\mathrm{Na}$ avaliação comportamental do perfil Psicoeducacional revisado observou-se maior déficit no quesito linguagem, enquanto na escala de desenvolvimento, o G\#1 executou com êxito a maior parte das atividades propostas. G\#2 não obteve êxito em toda as atividades que exigiam comunicação verbal. Na escala Vineland, ocorreu comprometimento na área de comunicação verbal nos gêmeos, enquanto as respostas de autonomia, socialização e motricidade foram satisfatórias. Embora o diagnóstico clínico esteja de acordo com o 
autismo, os resultados mostraram discordâncias quanto à condição clínica entre gêmeos. Conclusão. Estas diferenças podem estar relacionadas aos critérios médicos usados e fatores ambientais dispostos no momento das avaliações.

Unitermos. Autismo; Gêmeos univitelinos; Desenvolvimento; Comportamento; Linguagem

\begin{abstract}
Introduction. Childhood autism is part of a group of neurodevelopmental disorders described by Diagnostic and Statistical Manual of Mental Disorders 5 (DSM-5). Children with autistic disorder present functional impairments fundamental. Objective. To evaluate and describe aspects of behavioral development in univiteline twins (G\#1 e G\#2) with concordant medical diagnosis for autism, in the preschool stage. Method. A cross-sectional, prospective-described study. The data collection was performed through the application of the questionnaires CARS - Childhood Autism Rating Scale; Psychoeducational Revised Profile Scale; Vineland Adaptive Behavior Scale. Results. In CARS, G\#1 was classified as nonautistic and G\#2 a moderate autistic. In the behavioral evaluation of the revised Psychoeducational profile, the two children presented greater deficit in the language question, while in the development scale, the G\#1 successfully performed most of the proposed activities. G\#2 was disapproved in all activities that required verbal communication. On the Vineland scale, occurred verbal communication impairment in the twins, while the autonomy, socialization and motor responses were satisfactory. Although the clinical diagnosis agrees with autism the results of this research showed disagreements regarding the clinical condition between twins. Conclusion. These differences may be related to the medical criteria used and the environmental factors arranged at the time of the evaluations.
\end{abstract}

Keywords. Autism; Univiteline twins; Development; Behavior; Language

\title{
Resumen
}

Introducción. El autismo infantil es parte de un grupo de trastornos del desarrollo neurológico descritos por el Manual Diagnóstico y Estadístico de los Trastornos Mentales (DSM-5). Los niños con trastorno autista presentan deficiencias en tres áreas fundamentales: déficits en la variabilidad social, comunicativa y conductual: actividades restrictivas, repetitivas y estereotipadas. Objetivo. Avaluar y describir aspectos del desarrollo conductual de un caso de gemelos univitelinos (G\#1 e G\#2) con diagnóstico médico de autismo, en la etapa preescolar. Método. Estudio transversal, descriptivo prospectivo. La recolección de datos se realizó mediante la aplicación de los cuestionarios CARS - Escala de Calificación de Autismo Infantil; Escala de Perfil Revisada Psicoeducativa; Escala de Comportamiento Adaptativo de Vineland. Resultados. En CARS, G\#1 fue clasificado como no autista y G\#2 como autista moderado. En la evaluación conductual del perfil psicoeducativo revisado, los dos niños presentaron mayor déficit en las preguntas de lenguaje, mientras que en la escala de desarrollo, G\#1 realizó con éxito la mayoría de las actividades propuestas. G\#2 fue desaprobado en todas las actividades que requerían comunicación verbal. En la escala de Vineland, ocurrió un deterioro de la comunicación verbal en los gemelos, mientras que la autonomía, socialización y respuestas motoras fueron satisfactorias. Aunque el diagnóstico clinico concuerda con el autismo, los resultados de esta investigación mostraron desacuerdos con respecto a la condición clínica entre gemelos. Conclusión. Estas diferencias pueden estar relacionadas con los criterios médicos utilizados y los factores ambientales dispuestos en el momento de las evaluaciones.

Palabras clave: Autismo; Gemelos Univitelinos; Desarrollo; Comportamiento; Lenguaje

Trabalho realizado na Universidade Estadual de Ciências da Saúde de Alagoas (UNCISAL), Maceió-AL, Brasil.

Conflito de interesse: não $\quad$ Recebido em: 08/02/2021 Aceito em: 10/11/2021

Endereço para correspondência: José Claudio da Silva. Laboratório de Neurociência, Núcleo de Ciências Biológicas (NUCIB), Universidade Estadual de Ciências da Saúde de Alagoas (UNCISAL). Rua Dr. Jorge de Lima 113. Trapiche da Barra. Maceió-AL, Brasil. CEP 57010-300. Email: jcsneuroc1@gmail.com 


\section{INTRODUÇÃO}

O autismo apresenta características heterogêneas no neurodesenvolvimento e diferentes etiologias ${ }^{1}$. Os autistas compartilham comprometimentos com base em alterações neurológicas de áreas específicas do desenvolvimento tais como: déficits nas habilidades sociais de interação, habilidades comunicativas, verbais ou não-verbais afetadas, e uma variabilidade comportamental com atividades restritivas, repetitivas e estereotipadas ${ }^{2}$. Se apresenta topograficamente de modo muito heterogêneo e a diferenciação deste espectro se faz a partir da intensidade (leve, moderado e grave $)^{3}$.

Desde 1943 os questionamentos sobre a etiologia do autismo geram hipóteses que perpassam várias causas como neurobiológicas, anormalidades neuronais ou translocações cromossômicas, ou fatores socioculturais ${ }^{3,4}$. É um transtorno com evidências genéticas, pois gêmeos apresentam uma recorrência de 3 a $8 \%$ em famílias que já tenham uma criança autista. Em gêmeos monozigóticos, se usados critérios estritos se encontra um percentual importante para transtornos do espectro autista, o espectro de distúrbios de linguagem e socialização ${ }^{4,5}$.

O nosso objetivo foi avaliar e descrever a gravidade do autismo e características do desenvolvimento comportamental em gêmeos univitelinos, diagnosticados em fase pré-escolar. 


\section{MÉTODO}

Estudo de corte transversal e descrito, em gêmeos univitelinos, gênero masculino e diagnosticados por neurologista infantil (registro de CID F84.0 (F83).

Coleta de dados realizada mediante a aplicação única de questionários validados, após aprovação pelo Comitê de Ética em Pesquisa da UNCISAL sob o CAAE: 17073219.0.0000.5011 e assinatura dos Termos de Consentimento Livre e Esclarecido e Assentimento Livre e Esclarecido.

\section{Procedimento}

Instrumentos de coleta:

Childhood Autism Rating Scale (CARS): classifica a gravidade do autismo: leve, moderado e severo ${ }^{6,7}$; Escala de Perfil Psicoeducacional Revisto (PEP-R): descreve padrões de aprendizagem irregulares e idiossincráticos, coordenações e outras aquisições psicomotoras ${ }^{8,9}$. Usado para nortear a elaboração do planejamento Psicoeducacional, segundo o modelo de Treatment and Education of Autistic and Communication Handicapped Children, que é amplamente utilizada ${ }^{8-12}$. E a escala de Vineland que avalia Comportamentos Adaptativos ${ }^{13,14}$.

\section{RELATO DO CASO}

Os comportamentos autísticos das crianças foi percebido, primariamente no ambiente escolar, visto que, eram diferentes dos demais alunos. Comunicado aos pais, 
estes começaram a observar os filhos. A genitora começou a perceber que muitos dos comportamentos que as professoras relatavam e que ela também notara, posteriormente, convergiam com aquelas citados pelo profissional médico. A genitora relatou que não se preocupou porque pensou que os atrasos eram normais, pois o filho mais velho também apresentou. Questionados os pais sobre fatores precipitantes, relataram que descobriram a última gravidez após um acidente de moto, e que estava na quinta semana. Em todas as gestações, houve hipertensão arterial descompensada como principal intercorrência, e pré-eclampsia.

\section{Nascimento e desenvolvimento autístico}

Os gêmeos, G\#1 e G\#2, aos 3 anos no momento da avaliação, filhos de mãe multípara, parto gemelar cesariano e prematuro, aos sete meses de gestação. Ao nascer, os gêmeos ficaram dezesseis dias internados, oito em UTI neonatal usando surfactante. Apresentaram quadro de hiporreflexia ao nascer, padrão de sono regular, ruídos vocais enquanto dormiam e fez o desmame do leite materno fisiológico logo ao nascimento.

G\#2 apresentou mais dificuldade na alimentação quando comparado ao irmão (G\#1), padrão de sono inquieto, sialorreia intensa e bruxismo, reduzido controle dos esfíncteres urinários e do trato digestório. Os gêmeos eram intolerantes ao barulho, multidões e tinham medo de imagens sacras, motocicletas e água mesmo com idade de 
três anos, mas, após terapias diversas (psicológica, fisioterapêutica e terapia ocupacional) o quadro clínico melhorou. Apresentavam comportamentos de tiques não característicos da Síndrome de Gilles de La Tourette; lavavam as mãos com recorrência, polaciúria e bebiam água com certa frequência. G\#1 têm mãos inquietas.

Quanto ao desenvolvimento motor, ambos sustentaram a cabeça aos seis meses e deambularam aos 14 meses. O domínio de linguagem se desenvolveu com maior dificuldade, caracterizando-se disfásica em especial para G\#2. As primeiras palavras foram pronunciadas aos três anos, sem gagueira, ecolalia ou outro distúrbio. G\#1 possuía boa compreensão da linguagem falada, atendia ordens simples e imitava facilmente, enquanto $\mathrm{G \# 2}$ não apresentava este repertório, e evoluiu com disfasia de expressão e compreensão. Atividades psicomotoras preferidas: brinquedos de encaixe e organização de materiais de cores preferencialmente azul e amarelo.

As relações sociais na escola eram boas, mas em outros ambientes preferiam brincar por tempo muito prolongado. G\#1 mostrou maior facilidade para formar vínculo. Ambos realizavam tratamento com psicologia, fonoaudiologia, equoterapia e usam Neuleptil 3x ao dia.

Um processo de hospitalização de G\#2 afastou os gêmeos, trazendo algumas características interessantes para G\#1. Neste período, G\#1 desenvolveu várias habilidades que não apresentava na presença do irmão, como: melhora na linguagem, psicomotricidade e interação 
social; é possível que as inabilidades antes apresentadas por G\#1 estivessem atreladas a um processo de imitação dos comportamentos de G\#2.

\section{RESULTADOS}

A Tabela 1 discrimina as pontuações adquiridas no CARS para cada domínio nos comportamentos observados em cada um dos gêmeos, permitindo uma comparação diagnóstica tanto quantitativa quanto qualitativa.

Tabela 1. Diagnóstico pelo CARS de G\#1 e G\#2.

\begin{tabular}{l|cc}
\hline \multirow{2}{*}{ Domínios } & \multicolumn{2}{c}{ Pontos } \\
\cline { 2 - 3 } & G\#1 & G\#2 \\
\hline Relação com pessoas & 2 & 2 \\
Imitação & 2 & 4 \\
Resposta emocional & 3 & 3 \\
Uso corporal & 2 & 2 \\
Uso de objetos & 1 & 1 \\
Adaptação à mudanças & 2 & 2 \\
Resposta visual & 2 & 2 \\
Resposta auditiva ao som & 2 & 3 \\
Resposta ao paladar, olfato e tato & 1 & 1 \\
Medo ou ansiedade & 2 & 2 \\
Comunicação verbal & 2 & 4 \\
Comunicação não-verbal & 1 & 2 \\
Nível de atividade & 1 & 3 \\
Nível e consistência da resposta intelectual & 3 & 3 \\
Impressão global & 2 & $\mathbf{3 6}$ \\
\hline Cotação total & $\mathbf{2 8}$ & \\
\hline \hline
\end{tabular}


De acordo com os resultados da CARS (Tabela 1), evidencia-se que ao apresentar uma cotação total de 28 pontos contra 36, G\#1 é classificado como não autista e G\#2 como um autista ligeiramente moderado. A escala evidencia que maiores déficits em $\mathrm{G \# 1}$ se encontram nos domínios: resposta emocional moderadamente anormal com reações muito apagadas ou excessivas, e outras vezes não relacionadas com a situação; nível e consistência da resposta intelectual moderadamente anormal, onde a criança não é esperta para a idade. Entretanto, uma ou mais áreas podem funcionar próximo do normal.

Em G\#2, há seis áreas com maior comprometimento: imitação severamente anormal, onde a criança raramente imita ou nunca imita sons, palavras ou movimentos mesmo com ajuda. A resposta emocional desajustada com reações muito apagadas ou excessivas e as vezes não relacionada com a situação. As respostas auditivas eram muito variáveis, ignorando sons, tapando ouvidos, ou assustandose com sons do cotidiano. A comunicação verbal severamente comprometida, não utilizando a linguagem com sentido, reproduzia gritos, sons esquisitos ou complexos, simulando linguagem. Nível de atividade moderadamente anormal, muito ativo e difícil de conter; e nível de consciência da resposta intelectual moderadamente anormal, estando baixo para a idade, com uma ou mais áreas funcionando próximo do normal. 


\section{PEP-R}

Na escala de avaliação comportamental (Tabela 2), as duas crianças apresentaram maior déficit no quesito linguagem. G\#2 apresenta gravidade maior nas respostas, pontuando oito quesitos como grave, um moderado e apenas duas adequadas. Enquanto G\#1 apresenta duas respostas classificadas como gravemente deficitárias, três moderadas e seis adequadas. Nos demais quesitos apresentaram respostas adequadas ou moderadas.

Tabela 2. Escala Comportamental de G\#1 e G\#1.

\begin{tabular}{|c|c|c|c|c|c|c|}
\hline & \multicolumn{2}{|c|}{$\mathbf{A}$} & \multicolumn{2}{|c|}{$\mathbf{L}$} & \multicolumn{2}{|c|}{$\mathbf{S}$} \\
\hline & G\#1 & G\#2 & G\#1 & G\#2 & G\#1 & G\#2 \\
\hline Relação & 8 & 6 & - & 2 & - & - \\
\hline Materiais & 5 & 4 & 1 & 2 & - & - \\
\hline Sensorial & 6 & 6 & - & - & - & - \\
\hline Linguagem & 6 & 2 & 3 & 1 & 2 & 8 \\
\hline
\end{tabular}

$\mathrm{Na}$ escala de desenvolvimento (Tabela 3) G\#1 executou com êxito a maior parte das atividades, sendo reprovado ao não conseguir combinar 9 letras no cartão de letras e foi emergente nas atividades para nomear imagens e cores. G\#2 não realizou de forma adequada as atividades que exigiam comunicação verbal, e embora reconhecesse os objetos, não era capaz de nomeá-los, evidenciando ser seu maior déficit. 


\begin{tabular}{|c|c|c|c|c|c|c|}
\hline & \multicolumn{2}{|c|}{$\mathbf{P}$} & \multicolumn{2}{|c|}{ E } & \multicolumn{2}{|c|}{$\mathbf{F}$} \\
\hline & G\#1 & G\#2 & G\# 1 & G\#2 & G\#1 & G\#2 \\
\hline Imitação & 2 & 2 & 1 & 1 & - & - \\
\hline Percepção & 2 & 1 & - & - & - & 1 \\
\hline Motricidade fina & 1 & 1 & - & - & - & - \\
\hline Motricidade global & 2 & 2 & - & - & - & - \\
\hline Óculo-manual & 2 & 2 & - & - & 1 & 1 \\
\hline Realização & 7 & 4 & - & - & - & 3 \\
\hline Verbal & 1 & - & 3 & - & - & 4 \\
\hline
\end{tabular}

\section{Vineland}

$\mathrm{Na}$ área de comunicação, quatro respostas foram cotadas como nunca realizadas pelas crianças. Na área de autonomia, G\#2 apresentou seis pontos para tarefas que normalmente realiza, duas respostas cotadas como nunca realizam e quatro respostas que o avaliador não teve oportunidade de observar. No mesmo quesito, G\#1 apresentou um somatório de oito pontos para tarefas que normalmente realiza, uma resposta cotada como nunca realiza, em três respostas o avaliador não teve oportunidade de observar, e uma resposta cotada como desconhecida. Em socialização, ambos apresentaram uma resposta que normalmente realizam e três que nunca realizam. Na área de motricidade, os gêmeos apresentaram igualmente um somatório de seis respostas que normalmente realizam, três que nunca realizam e sete 
respostas que o avaliador não teve oportunidade de observar.

\section{DISCUSSÃO}

Observa-se que a área de comunicação verbal de ambos se encontra acentuadamente comprometida, uma vez que foram incapazes de apresentar respostas satisfatórias para questionamentos que normalmente crianças da mesma faixa etária responderiam com facilidade. Isto corrobora descrições da literatura em que gêmeos autistas apresentam comportamentos que não são tipicamente observados em neurotípicos ${ }^{15}$. Quanto a autonomia, socialização e motricidade, o desenvolvimento foi satisfatório em quase sua totalidade nos gêmeos de acordo com os resultados obtidos e demonstrados neste trabalho; isto também corrobora com relatos que são descritos pela literatura nacional e internacional ${ }^{16}$.

Foi possível conhecer fatores importantes que podem ter influenciado o desenvolvimento do comportamento autístico nos gêmeos, pois, há fatores e sintomatologias que são predominantes como condições amplamente percebidas que são os distúrbios de linguagem e de socialização. Os trabalhos realizados fomentam que há sintomatologias em casos de autismo em gêmeos característicos e predominantes, os quais contribuem para percepção deste transtorno ${ }^{17,18}$. E que o conjunto de fatores observados e associados com as ferramentas avaliativas, se 
tornam instrumentos imprescindíveis e corroborativos para facilitação diagnóstica, sempre sendo fortalecido pelo fallow-up dos casos.

A literatura internacional discute possíveis mecanismos associados a origem e o desenvolvimento do Transtorno do Espectro Autismo. Um trabalho retrospectivo realizado com prontuários de 75 crianças autistas demonstrou uma possível associação entre o autismo e diversos fatores de riscos ${ }^{19}$. Foi observado que parto prematuro, asfixia perinatal e baixo peso ao nascer, além de alterações nos achados imaginológicos de exames de Ressonância Nuclear Magnética (RNM) demonstrado em 25,3\% da amostra ( $n=19$ crianças). Este estudo demostra uma ligação dos fatores de risco observados nos gêmeos autistas descritos neste relato de caso.

Entretanto, há trabalhos demonstrando que alterações genéticas estão diretamente relacionadas ao desenvolvimento de autismo em gêmeos como descreveu ${ }^{20-}$ 22. Além disto pesquisadores defendem outros paradigmas como principais causas do desenvolvimento do transtorno como biológicos-genéticas, relacional, ambiental e até mesmo uma condição de neurodiversidade ${ }^{23}$.

Mesmo havendo semelhanças nas anamneses realizadas nos casos de autismo, nós demonstramos que ainda há dificuldades no diagnóstico quando as avaliações são realizadas para a detecção em gêmeos. Mesmo a síndrome de Asperger quando atinge gêmeos monozigóticos são diagnósticadas ou tratadas 
tardiamente ${ }^{24}$. Mas a literatura relata que traços autistas podem ser apresentar capacidades de cognição social reduzidas predominantemente em gêmeos do sexo masculino, apesar de uma extensão comparável de traços autistas em cada $\operatorname{sexo}^{25}$. O estudo demonstrou de forma consistente, associações entre cognição social e a estrutura do social do cérebro. Foi observado que a menor capacidade cognitiva foi associada com o aumento da espessura do córtex cerebral em diferentes regiões do cérebro, e mais notadamente no sexo masculino ${ }^{25-27}$.

Estudos sugerem que o uso de exames de imagens como a RNM são fundamentais em investigações iniciais para auxiliar no diagnóstico de autismo em gêmeos, principlamente, quando os casos atingem o sexo masculino. Foi demonstrado que os achados incidentais assintomáticos em imagens podem incluir fatores ambientais e genéticos em autistas. Houve presença de achados incidentais em $68 \%$ dos casos de transtornos do especto autistas em gemelares, representando uma quantidade maior quando comparados a filhos únicos com autismo ${ }^{28}$. E que isto pode ser resultado do ambiente de desenvolmento fetal que é compartilhado por gêmeos, ainda dentro do útero materno. O incremento de exames de imagens como a RNM pode ser uma ferramenta importante para auxiliar no fechamento do diagnóstico correto e precoce.

Mesmo havendo crianças autistas do mesmo ambiente familiar, a evolução fisiopatológica apresenta-se de forma diferenciada e/ou em estágios de evolução distintos. Isto 
requer uma atenção que pode ser de maneira mais detalhada e cautelosa, mesmo entre gemelares univitelinos.

\section{CONCLUSÃO}

Os dados colhidos podem ser usados para nortear possíveis planos de estudos futuros neste grupo importante de pessoas que tenha transtorno do espectro autista, assim como na Síndrome de Asperger.

\section{REFERÊNCIAS}

1.Zafeiriou DI, Ververi A, Vargiami E. Childhood autism and associated comorbidities. Brain Dev 2007;29:257-72. http://dx.doi.org/10.1016/j.braindev.2006.09.003

2.Griesi-Oliveira K, Sertié AL. Autism spectrum disorders: an updated guide for genetic counseling. Einstein 2017;15:233-8. http://dx.doi.org/10.1590/S1679-45082017RB4020

3.Xu W, Yao J, Liu W. Intervention Effect of Sensory Integration Training on the Behaviors and Quality of Life of Children with Autism. Psychiatr Danub http://dx.doi.org/10.24869/psyd.2019.340

4. Fakhro KA. Genomics of Autism. Adv Neurobiol 2020;24:83-96. http://dx.doi.org/10.1007/978-3-030-30402-7 3

5.Saffari A, Arno M, Nasser E, Ronald A, Wong CCY, Schalkwyk LC, et al. RNA sequencing of identical twins discordant for autism reveals blood-based signatures implicating immune and transcriptional dysregulation. Mol Autism 2019;7:38. http://dx.doi.org/10.1186/s13229-019-0285-1

6.Pereira A, Riesgo RS, Wagner MB. Childhood autism: translation and validation of the Childhood Autism Rating Scale for use in Brazil. J Pediatr 2008;84:487-94. https://doi.org/10.1590/S002175572008000700004

7.Grissom M. Childhood Autism Rating Scales. In: Kreutzer JS, DeLuca J, Caplan B. (eds). Encyclopedia of Clinical Neuropsychology. California: Springer; 2011. https://doi.org/10.1007/978-0-387-79948-3 1530

8.Schopler E, Mesibov G, Baker A. Evaluation of Treatment for Autistic Children and Their Parents. J Am Acad Child Psychiatr 1982;21:262-7. https://doi.org/10.1016/S0002-7138(09)60881-5 
9.Leon V, Bosa C, Hugo C, Hutz CS. Psychometric Properties of the Psychoeducational Profile Revised: PEP-R. Aval Psicol 2004;3:39-52. http://pepsic.bvsalud.org/scielo.php?script=sci arttext\&pid=S1677-

$04712004000100005 \& \mathrm{lng}=\mathrm{pt \& nrm}=$ iso\&tlng=pt\#enderb

10.Gillberg C. Autism and pervasive developmental disorders. J Child Psychol Psychiatr 1990;31:99-119. https://doi.org/10.1111/j.14697610.1990.tb02275.x

11.Antão JYFL, Oliveira ASB, Barbosa RTA, Crocetta TB, Guarnieri R, Arab $\mathrm{C}$, et al. Instruments for augmentative and alternative communication for children with autism spectrum disorder: a systematic review. Clinics 2018;73:e497. http://dx.doi.org/10.6061/clinics/2017/e497

12. Muris $P$, Steerneman $P$, Ratering $E$. Brief report: Interrater reliability of the Psychoeducational Profile (PEP). J Autism and Dev Disord 1997;27:621-6. https://doi.org/10.1023/A:1025838312457

13.Sparrow SS, Cicchetti DV. Diagnostic uses of the Vineland Adaptive Behavior Scales. J Ped Psychol 1985;10:21525. http://dx.doi.org/10.1093/jpepsy/10.2.215

14.Carter AS, Volkmar FR, Sparrow SS, Wang JJ, Lord C, Dawson G, et al. The Vineland Adaptive Behavior Scales: Supplementary Norms for Individuals with Autism. J Autism Dev Disord 1998;28:287-302. https://doi.org/10.1023/A:1026056518470

15. Coutinho JVSC, Bosso RMV. Gêmeos dizigóticos concordantes para autismo: relato de caso. Rev Bras Neurol Psiqu 2016;20:158-69. https://www.revneuropsiq.com.br/rbnp/article/view/102/98

16.Geschwind DH. Autism: Many Genes, Common Pathways. Cell 2008;135:391-5. https://doi.org/10.1016/j.cell.2008.10.016

17.Gadia CA, Tuchman R, Rotta NT. Autismo e doenças invasivas de desenvolvimento. J Ped 2004;80:12. https://doi.org/10.1590/S002175572004000300011

18.Solís-Añes E, Delgado-Luengo W, Hernández ML. Autismo, cromosoma 15 y la hipótesis de disfunción GABAérgica. Revisión Invest Clin 2007; 48:529-41.

http://ve.scielo.org/pdf/ic/v48n4/art12.pdf

19.Fezer GF, Matos MB, Nau AL, Zeigelboim BS, Marques JM, Liberalesso PBN. Perinatal features of children with autism spectrum disorder. Rev Paul Pediatr 2017;35:130-5. http://dx.doi.org/10.1590/1984-0462/;2017;35;2;00003

20.Tick B, Bolton P, Happe F, Rutter M, Rijsdijk F. Heritability of autism spectrum disorders: a meta-analysis of twin studies. J Child Psychol Psychiatr 2016;57:585-95. http://dx.doi.org/10.1111/jcpp.12499

21. Hegarty II JP, Lazzeroni LC, Raman MM, Hallmayer JF, Cleveland SC, Wolke ON, et al. Genetic and environmental influences on corticostriatal circuits in twins with autism. J Psychiatry Neurosci 2020;45:188-97. http://dx.doi.org/10.1503/jpn.190030

22.Imamura A, Morimoto $\mathrm{Y}$, Ono S, Kurotaki N, Kanegae S, Yamamoto $\mathrm{N}$, et al. Genetic and environmental factors of schizophrenia and autism spectrum disorder: insights from twin 
studies

https://doi.org/10.1007/s00702-020-02188-w

23.Fadda GM, Cury VE. O enigma do autismo: contribuições sobre a etiologia do transtorno. Psicol Estudo 2016;21:411-23. http://dx.doi.org/10.4025/psicolestud.v21i3.30709

24.Costa TF, Marega RS, Vidotti MZ. Síndrome de Asperger em gêmeos monozigóticos: Relato de caso. Rev Cient Multidiscipl Núcleo do

http://dx.doi.org/10.32749/nucleodoconhecimento.com.br/saude/sin drome-de-asperger

25.Cauvet É, Van't Westeinde A, Toro R, Kuja-Halkola R, Neufeld J, Mevel $\mathrm{K}$, et al. The social brain in female autism: a structural imaging study of twins. Soc Cogn Affect Neurosci 2020;15:423-36. http://dx.doi.org/10.1093/scan/nsaa064

26. Hull L, Mandy W, Petrides KV. Behavioural and cognitive sex/gender differences in autism spectrum condition and typically developing males and females. Autism Inter J Res Pract 2017;21:706-27. http://dx.doi.org/10.1177/1362361316669087

27.Isaksson J, Van't Westeinde A, Cauvet É, Kuja-Halkola R, Lundin $\mathrm{K}$, Neufeld J, et al. Social Cognition in Autism and Other Neurodevelopmental Disorders: A Co-twin Control Study. J Autism Dev Disord 2019;49:2838-48. http://dx.doi.org/10.1007/s10803019-04001-4

28. Monterrey JC, Philips J, Cleveland S, Tanaka S, Barnes P, Hallmayer JF, et al. Incidental brain MRI findings in an autism twin study. Autism

Res 2017,10:113-20. https://doi.org/10.1002/aur.1720 